\title{
YAP1 promotes high glucose-induced inflammation and extracellular matrix deposition in glomerular mesangial cells by modulating NF-кB/JMJD3 pathway
}

\author{
YAN WANG, JINMEI XU and ZHIFENG CHENG \\ Department of Endocrinology, Fourth Affiliated Hospital of Harbin Medical University, \\ Harbin, Heilongjiang 150001, P.R. China
}

Received November 25, 2020; Accepted July 2, 2021

DOI: $10.3892 /$ etm.2021.10784

\begin{abstract}
Diabetic nephropathy (DN) is one of the most serious microvascular complications of late-stage diabetes. Glomerular mesangial cell (GMC) proliferation and excessive extracellular matrix (ECM) deposition are the main pathological characteristics associated with the occurrence and development of DN. Yes-associated protein 1 (YAP1) can bind to several transcription factors and is associated with the development of various diseases. However, the effects of YAP1 on DN remain unclear. The aim of the present study was to explore the regulatory effect and potential mechanism of YAP1 in glucose-induced inflammation and ECM deposition in high-glucose-treated GMCs. In the present study, HBZY-1 cell models treated with high glucose were constructed, and the effects of YAP1 on the proliferation, inflammation, ECM deposition and fibrosis of HBZY-1 cells were detected. The results showed that YAP1 was highly expressed in HBZY-1 cells treated with high glucose and that YAP1 silencing decreased cell viability, the levels of inflammatory cytokines, ECM deposition and the degree of fibrosis in cells. Further experiments revealed that $\mathrm{NF}-\kappa \mathrm{B} / \mathrm{Jumonji}$ domain-containing protein D3 (JMJD3) signaling pathway inhibitors alleviated the promoting effect of YAP1 overexpression on inflammatory response and ECM deposition in HBZY-1 cells treated with high glucose. In conclusion, it was demonstrated that YAP1 can promote high glucose-induced inflammation and ECM deposition by activating the $N F-\kappa B / J M J D 3$ signaling pathway in GMCs.
\end{abstract}

Correspondence to: Dr Zhifeng Cheng, Department of Endocrinology, Fourth Affiliated Hospital of Harbin Medical University, 37 Yiyuan Street, Nangang, Harbin, Heilongjiang 150001, P.R. China

E-mail:msihxioxiao@163.com

Key words: diabetic nephropathy, YAP1, glomerular mesangial cells, inflammation, extracellular matrix deposition, NF-кB/JMJD3

\section{Introduction}

Diabetic nephropathy (DN) is a microvascular complication of diabetes that represents the leading cause of mortality among patients with type 1 diabetes mellitus (DM) and the second leading cause of mortality among those with type 2 DM. DN is also the primary cause of end-stage renal disease (ESRD) $(1,2)$. Therefore, studying the mechanism of DN pathogenesis and controlling its progression is urgently required.

The pathogenesis of DN is complex and includes hemodynamic changes, glucose and lipid metabolism disorders, autophagy, inflammation and growth factor disorders (3-5). Mesangial cells are important inherent cells in the glomerulus which, together with the extracellular matrix (ECM) they secrete, constitute the glomerular mesangial region and play an important role in supporting the capillary loop $(6,7)$. In glomerular diseases, mesangial cells are one of the most important effector cells involved in the disease process. Mesangial cells can promote cell proliferation and ECM deposition by auto-activation and secretion of several bioactive molecules to affect the function of other inherent cells in the glomeruli through paracrine mechanisms, in turn participating in the process of glomerular injury or repair and ultimately promoting the occurrence and development of glomerulosclerosis $(8,9)$. Therefore, exploring the mechanism of abnormal mesangial cell function in diabetes will provide a reliable theoretical basis for developing a more effective therapeutic approach.

Yes-associated protein 1 (YAP1) is an oncogenic transcriptional co-regulator and the effector molecule of a variety of signaling pathways. The expression level of YAP1 was found to be higher in activated fibroblasts. Moreover, YAP1 may promote the activation of fibroblasts and participate in the secretion of ECM by regulating the expression of activation-associated genes, including growth factors $(10,11)$. Podocyte injury has been reported to stimulate the activity of transcriptional coactivator YAP and the expression of the YAP target gene in a rat glomerular disease model; in addition, YAP overexpression increased the levels of ECM-associated proteins, which may lead to fibrosis (12). In a different study, Chen et al (13) found that YAP1 knockdown 
inhibited the deposition of ECM and ultimately improved pulmonary fibrosis in vitro and in vivo. In addition, YAP1 has been proven to promote tumorigenesis in vivo, in vitro and in human specimens. YAP1 may activate the transcription of the inflammatory cytokine IL- $1 \beta$ and induce the development of inflammation-associated gastric cancer; therefore, YAP1 may activate inflammatory cytokines to further aggravate the inflammatory response (14). However, the effect of YAP1 on a glomerular mesangial cell (GMC) model of DN treated with high glucose has not yet been studied in detail.

In the present study, the regulatory effect of YAP1 on mesangial cells treated with high glucose and the potential underlying mechanism were explored. The results revealed that YAP1 promoted inflammation and ECM deposition by activating the $\mathrm{NF}-\kappa \mathrm{B} / \mathrm{JMJD} 3$ signaling pathway in high glucose-induced GMCs. The findings of the present study may provide a new research direction for the diagnosis and treatment of DN.

\section{Materials and methods}

Cell culture and treatment. The HBZY-1 renal mesangial cell line was purchased from the American Type Culture Collection. HBZY-1 cells were cultured in $5.5 \mathrm{mmol} / 1 \mathrm{DMEM}$ (Cytiva) containing 10\% fetal bovine serum (Cytiva) in a humidified atmosphere of $95 \% \mathrm{O}_{2}$ and $5 \% \mathrm{CO}_{2}$ at $37^{\circ} \mathrm{C}$. Cells were not used beyond passage 3 . HBZY-1 cells cultured in $5.5 \mathrm{mmol} / 1$ serum-free DMEM normal-glucose media were used as the normal control group (NG) and cells cultured in $30 \mathrm{mmol} / 1$ serum-free DMEM high-glucose media were used as the high-glucose group (HG). Mannitol was used as a control to eliminate the effect of osmotic pressure. The model cells were treated with $30 \mathrm{mmol} / 1$ serum-free DMEM for 0,6 , 12,24 and $48 \mathrm{~h}$.

Cell transfection. The YAP1 overexpression vector pcDNA-TRIM31 (Oe-YAP1) and empty control vector pcDNA (Oe-NC) were constructed by Shanghai GenePharma Co., Ltd. Small interfering RNA (siRNA) against YAP1 (si-YAP1) and siRNA negative control (si-NC) were synthesized by Shanghai GenePharma Co., Ltd. A final concentration of $100 \mathrm{nM}$ plasmids were transfected into cells using Lipofectamine ${ }^{\circledR} 2000$ (Thermo Fisher Scientific, Inc.) transfection reagent. After $48 \mathrm{~h}$ transfection, the transfection efficiency was detected and cells were collected for subsequent experiments.

Cell counting kit-8 (CCK-8) assay. Cells were inoculated into 96-well plates $\left(5 \times 10^{3}\right.$ cells/well) during the logarithmic growth phase and 3 repeated wells were set. CCK-8 solution $(10 \mu \mathrm{l})$ was added to each well, and cells were incubated at $37^{\circ} \mathrm{C}$. The absorbance value in each well was detected at $450 \mathrm{~nm}$ at $72 \mathrm{~h}$ using a microplate reader (BioTek Instruments, Inc.).

Enzyme-linked immunosorbent assay (ELISA). The levels of tumor necrosis factor (TNF)- $\alpha$, interleukin (IL)-1 $\beta$ and IL-6 in each group were determined by ELISA performed using respective ELISA detection kits (cat. no. EK0526, EK0393 and EK0412; Wuhan Boster Biological Technology, Ltd.), according to the manufacturer's instructions.
Immunofluorescence. Immunofluorescence was used to detect the expression level of collagen IV in HBZY-1 cells. The glass slides containing HBZY-1 cells were washed by PBS twice, and then fixed with $4 \%$ paraformaldehyde for $10 \mathrm{~min}$ at room temperature. Next, cells were permeabilized with $0.5 \%$ Triton $\mathrm{X}-100$ for $10 \mathrm{~min}$ at room temperature and sealed with $5 \% \mathrm{BSA}$ for $30 \mathrm{~min}$ at room temperature. Primary antibody against collagen IV (cat. no. Ab6586; dilution, 1:1,000; Abcam) was added to the slide and incubated overnight at $4^{\circ} \mathrm{C}$. Finally, the nucleus was stained with DAPI solution at room temperature for $10 \mathrm{~min}$, and the stained sections were examined. Images were captured at x200 magnification under a light microscope (Olympus Corporation).

Reverse transcription-quantitative PCR (RT-qPCR). Total RNA was extracted from cells using TRIzol ${ }^{\circledR}$ reagent (Thermo Fisher Scientific, Inc.). RNA was then reverse-transcribed into cDNA using the QuantiTect Reverse Transcription kit (Qiagen $\mathrm{AB}$ ) and the reaction was incubated at $25^{\circ} \mathrm{C}$ for $5 \mathrm{~min}, 42^{\circ} \mathrm{C}$ for $30 \mathrm{~min}, 85^{\circ} \mathrm{C}$ for $5 \mathrm{~min}$ and then kept at $4^{\circ} \mathrm{C}$ for $5 \mathrm{~min}$. The PCR reaction was performed using the ABI 7500 Real Time PCR Detection System (ABI) following the Quantitect SYBR Green PCR kit protocols (Qiagen AB). The relative expression of target genes was calculated using the $2^{-\Delta \Delta C q}$ method (15). GAPDH was used as the internal control. The primer sequences were as follows: Yap1 forward, 5'-GCAACT CCAACCAGCAGCAACA-3'; Yap1 reverse, 5'-CGCAGC CTCTCCTTCTCCATCTG-3'; monocyte chemoattractant protein-1 (MCP-1) forward, 5'-ATAGCAGCCACCTTCATT CG-3'; MCP-1 reverse, 5'-TTCCCCAAGTCTCTGTATCT-3'; $\beta$-actin forward, 5'-TACATGGCTGGGGTGTTGAA-3'; and $\beta$-actin reverse 5'-AAGAGAGGCATCCTCACCCT-3'.

Western blot analysis. Total protein from the cells was extracted using RIPA lysis buffer (Thermo Fisher Scientific, Inc.). The protein concentration was determined using BCA protein quantitative kit (Haimen Biyuntian Biological Technology Co., Ltd.). Next, protein (30 $\mu \mathrm{g}$ per lane) was separated using $12 \%$ SDS-PAGE and then transferred to a PVDF membrane. These membranes were blocked with 5\% skimmed milk for $1 \mathrm{~h}$ at room temperature and incubated with primary antibodies against YAP1 (cat. no. ab52771; dilution, 1:1,000), proliferating cell nuclear antigen (PCNA; cat. no. ab29; dilution, 1:1,000), MCP-1 (cat. no. ab214819; dilution, 1:1,000), collagen IV (cat. no. ab6586; dilution, $1: 1,000)$, $\alpha$-smooth muscle actin ( $\alpha$-SMA; cat. no. ab240678; dilution, 1:1,000), fibronectin (FN; cat. no. ab6328; dilution, 1:1,000), connective tissue growth factor (CTGF; cat. no. ab209780; dilution, 1:1,000), TGF- $\beta 1$ (cat. no. ab215715; dilution, 1:1,000), phosphorylated inhibitor of

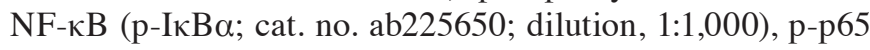
(cat. no. ab31624; dilution, 1:1,000), p-65 (cat. no. ab32536; dilution, 1:1,000), Jumonji domain-containing protein D3 (JMJD3; cat. no. ab38113; dilution, 1:1,000) and GAPDH (cat. no. ab9485; dilution, 1:1,000) overnight at $4^{\circ} \mathrm{C}$ (all from Abcam). Subsequently, these membranes were incubated with the corresponding mouse anti-rabbit and goat anti-mouse IgG secondary antibodies conjugated to horseradish peroxidase (sc-2357 and sc-2005, respectively; dilution, 1:5,000; Santa Cruz Biotechnology, Inc.) for $2 \mathrm{~h}$ at room temperature. Immunoreactive protein bands were visualized using an 

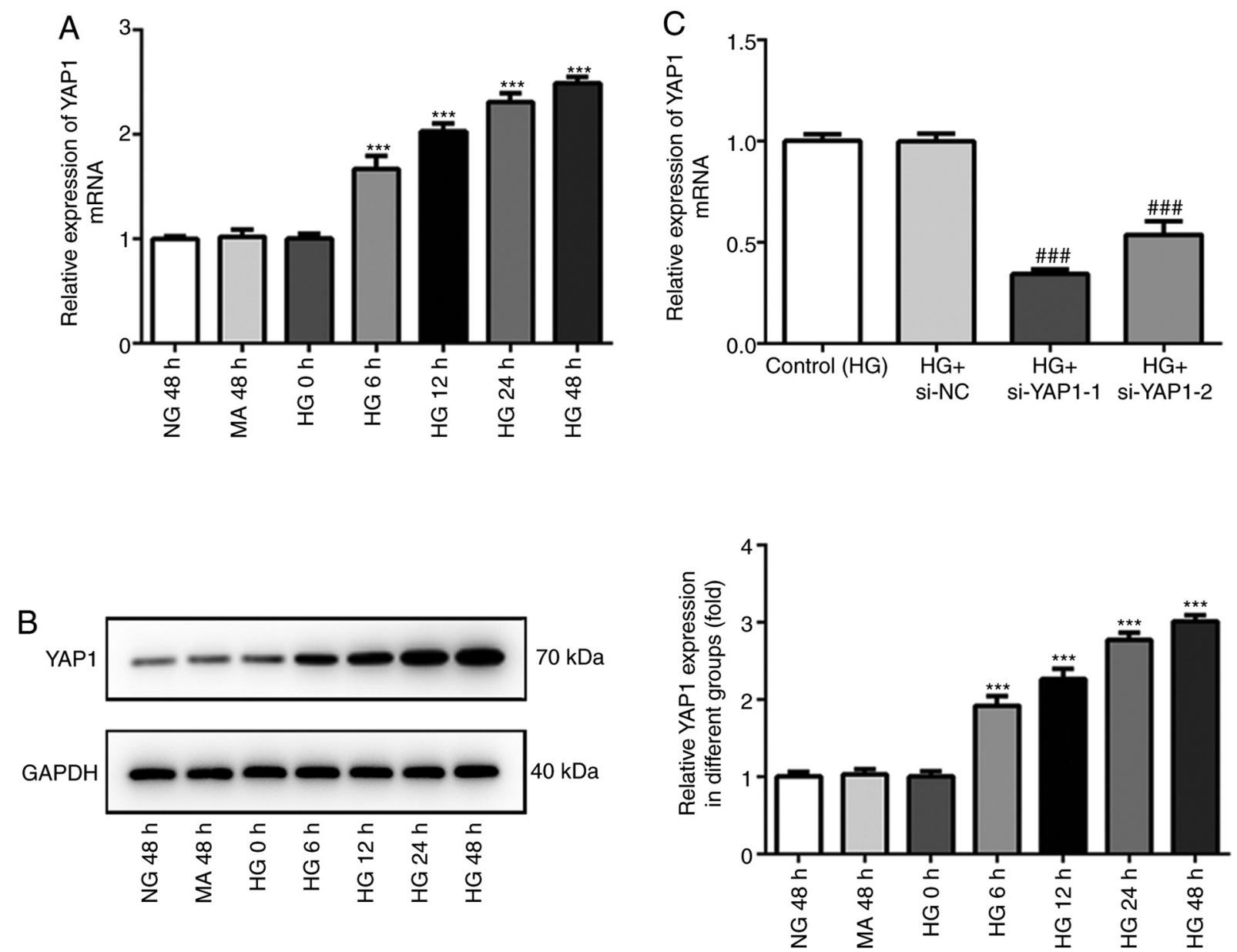

Figure 1. YAP1 is upregulated in HBZY-1 cells treated with high glucose. (A) RT-qPCR was used to measure the expression of YAP1 in HBZY-1 cells treated with $30 \mathrm{mmol} / 1 \mathrm{serum}$-free DMEM medium for 0, 6, 12, 24 and $48 \mathrm{~h}$, respectively. (B) Western blotting was used to measure the expression of YAP1 in HBZY-1 cells treated with $30 \mathrm{mmol} / 1 \mathrm{serum}$-free DMEM medium for 0, 6, 12, 24 and $48 \mathrm{~h}$, respectively. (C) Transfection efficiency of YAP1 small interfering RNA was detected by RT-qPCR. ${ }^{* * *} \mathrm{P}<0.001$ vs. HG 0 h; ${ }^{\# \# \# ~} \mathrm{P}<0.001$ vs. HG + si-NC. YAP1, Yes-associated protein 1; RT-qPCR, reverse transcription-quantitative PCR; si-, small interfering RNA; NC, negative control; HG, high-glucose group; NG, normal control group; MA, mannitol.

ECL kit (EMD Millipore) and quantified using densitometry (QuantityOne 4.5.0 software; Bio-Rad Laboratories, Inc.). GAPDH was used as an internal control.

Statistical analysis. Experimental data were analyzed using the SPSS 20.0 software (IBM Corp.), and the results are expressed as the mean \pm SEM. Unpaired, two-tailed Student's t-test was performed for comparisons between two groups. One-way analysis of variance test followed by Dunnett's post hoc test was performed for comparisons among multiple groups. $\mathrm{P}<0.05$ was considered to indicate a statistically significant difference.

\section{Results}

YAP1 is upregulated in HBZY-1 cells treated with high glucose. In order to study the role and mechanism of action of YAP1 in a cell model of DN treated with high glucose, cells were treated with $30 \mathrm{mmol} / 1$ serum-free DMEM high-glucose media. As shown in Fig. 1A and B, the RT-qPCR and western blot analysis results showed that YAP1 expression was increased in a time-dependent manner. YAP1 expression peaked at $48 \mathrm{~h}$. Therefore, the cell treatment time was set as $48 \mathrm{~h}$. In addition,
YAP1 expression in cells was decreased following transfection with YAP1 siRNA. As shown in Fig. 1C, compared with the $\mathrm{HG}+$ si-NC group, YAP1 expression was the lowest in the HG + si-YAP1-1 group. Thus, si-YAP1-1 was used for the subsequent experiments.

YAP1 knockdown inhibits the proliferation of HBZY-1 cells treated with high glucose. CCK-8 assay and western blot analysis were used to determine the effect of YAP1 on cell proliferation. The CCK-8 results showed that the cell viability in the HG + si-YAP1-1 group was significantly lower compared with that in the $\mathrm{HG}+$ si-NC group, indicating that YAP1 knockdown significantly decreased the proliferation of HBZY-1 cells treated with high glucose (Fig. 2A). In addition, western blot analysis results showed that YAP1 knockdown significantly decreased the expression of proliferation-associated protein PCNA (Fig. 2B).

YAP1 knockdown suppresses the inflammation of HBZY-1 cells treated with high glucose. Next, the expression level of inflammatory cytokines was detected by ELISA. As shown in Fig. 3A, the ELISA results showed that YAP1 knockdown significantly decreased the expression of the pro-inflammatory 

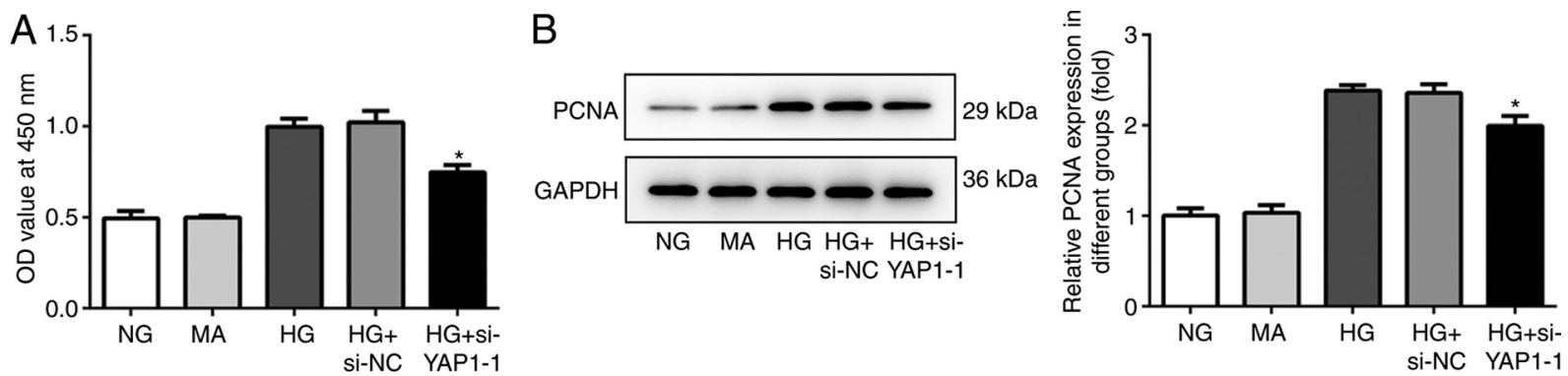

Figure 2. Knockdown of YAP1 inhibited the proliferation of HBZY-1 cells induced by high glucose. (A) Effect of YAP1 knockdown on the viability of HBZY-1 cells treated with high glucose was detected by Cell Counting Kit-8 assay. (B) Effect of YAP1 knockdown on the expression of PCNA in the HBZY-1 cells treated with high glucose was measured by western blotting. "P<0.05 vs. HG + si-NC. YAP1, Yes-associated protein 1; PCNA, proliferating cell nuclear antigen; si-, small interfering RNA; NC, negative control; HG, high-glucose group; NG, normal control group; MA, mannitol.
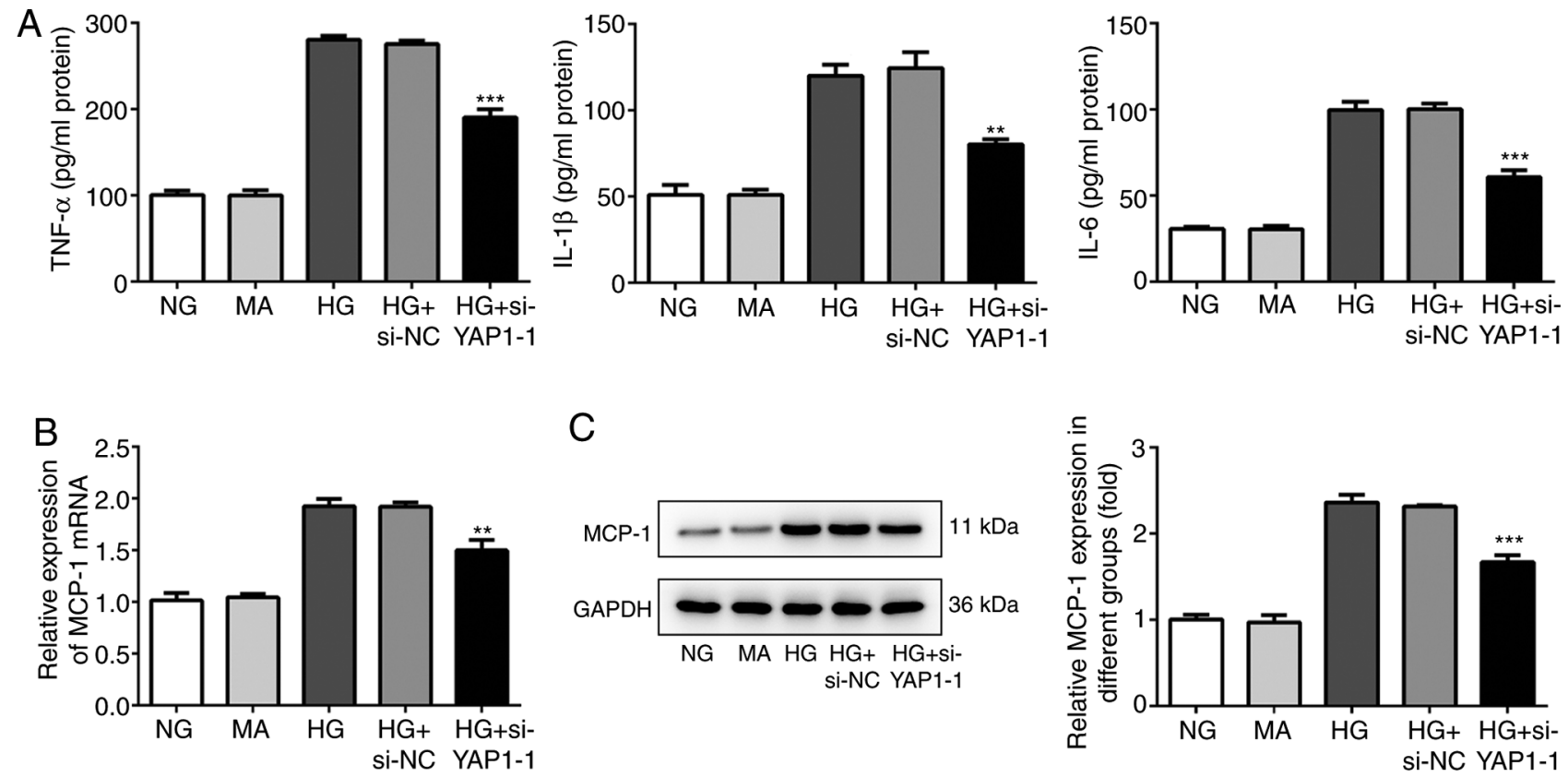

C
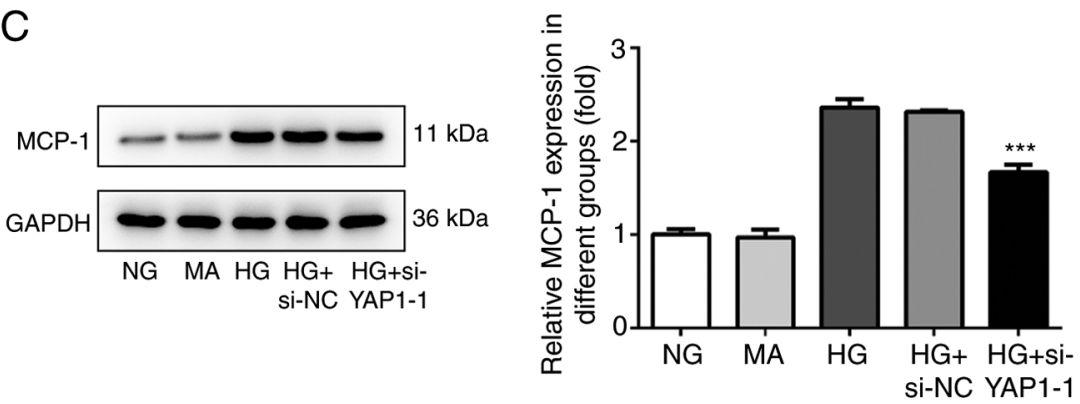

Figure 3. Knockdown of YAP1 suppresses the inflammation of HBZY-1 cells induced by high glucose. (A) Effect of YAP1 knockdown on the expressions of pro-inflammatory factors (TNF- $\alpha$, IL-1 $\beta$ and IL-6) was detected by enzyme-linked immunosorbent assay. (B) Effect of YAP1 knockdown on the expression of MCP-1 was detected by reverse transcription-quantitative PCR. (C) Effect of YAP1 knockdown on the expression of MCP-1 was detected by western blotting. ${ }^{* *} \mathrm{P}<0.01$ and ${ }^{* * *} \mathrm{P}<0.001$ vs. HG + si-NC. YAP1, Yes-associated protein 1; si-, small interfering RNA; NC, negative control; HG, high-glucose group; NG, normal control group; MA, mannitol; TNF, tumor necrosis factor; IL, interleukin; MCP-1, monocyte chemoattractant protein-1.

factors TNF- $\alpha$, IL- $1 \beta$ and IL-6. In addition, MCP-1, as an important inflammatory mediator, was detected by RT-qPCR and western blot analyses. As shown in Fig. 3B and C, YAP1 knockdown significantly decreased MCP-1 expression and then enhanced the inflammation of HBZY-1 cells treated with high glucose compared with the control group.

YAP1 knockdown inhibits ECM deposition and fibrosis in HBZY-1 cells treated with high glucose. Since the YAP1 inhibitor could significantly inhibit the deposition of ECM and pulmonary fibrosis (11), the effect of YAP1 on ECM deposition and fibrosis in HBZY-1 cells treated with high glucose was analyzed by western blot analysis and immunofluorescence. As shown in Fig. 4A, compared with the control group, YAP1 knockdown significantly decreased the expression of Leydig cell markers, including collagen IV, $\alpha$-SMA and FN. The expression of collagen IV was determined by immunofluorescence, and the results showed that YAP1 knockdown significantly decreased the fluorescence intensity of collagen IV (Fig. 4B and C). In addition, compared with the control group, YAP1 knockdown significantly decreased the expression of the fibrogenic factors CTGF and TGF- $\beta 1$ (Fig. 4D). In general, YAP1 knockdown could significantly enhance ECM deposition and fibrosis in HBZY-1 cells treated with high glucose.

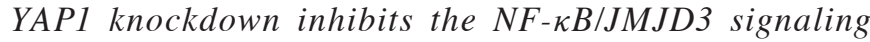
pathway in HBZY-1 cells treated with high glucose. To further study the mechanism of YAP1 on the HBZY-1 cell injury induced by high glucose, the effect of YAP1 on the activation of the NF- $\kappa \mathrm{B} / \mathrm{JMJD} 3$ signaling pathway was detected by western blot analysis. As shown in Fig. 5A and B, YAP1 knockdown significantly inhibited the phosphorylation levels of $\mathrm{I} \kappa \mathrm{B} \alpha$ and p65, and decreased JMJD3 expression. These results suggested 
A

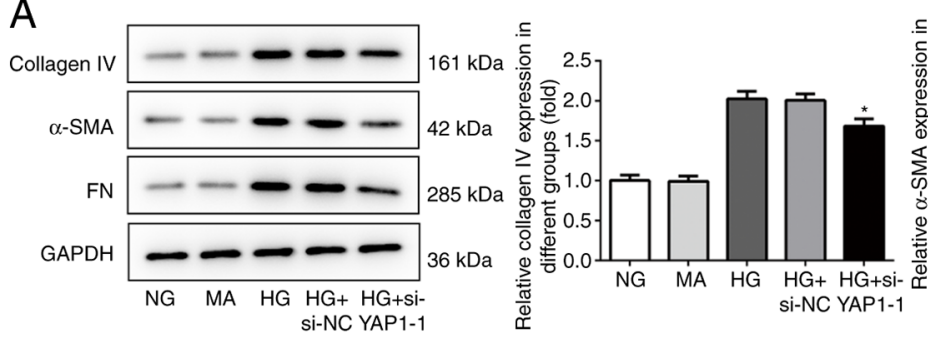

B
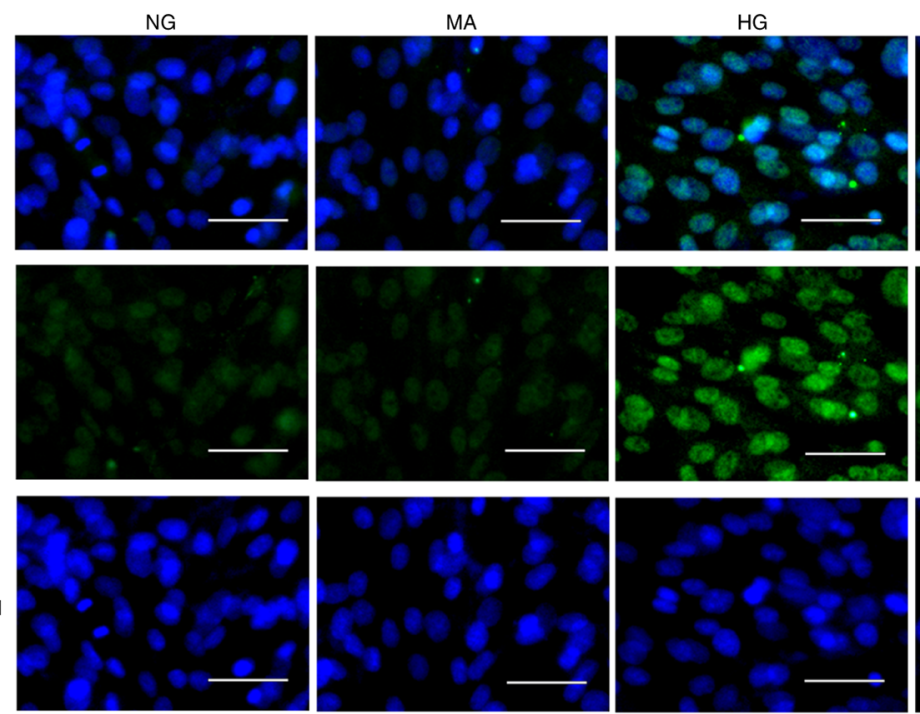
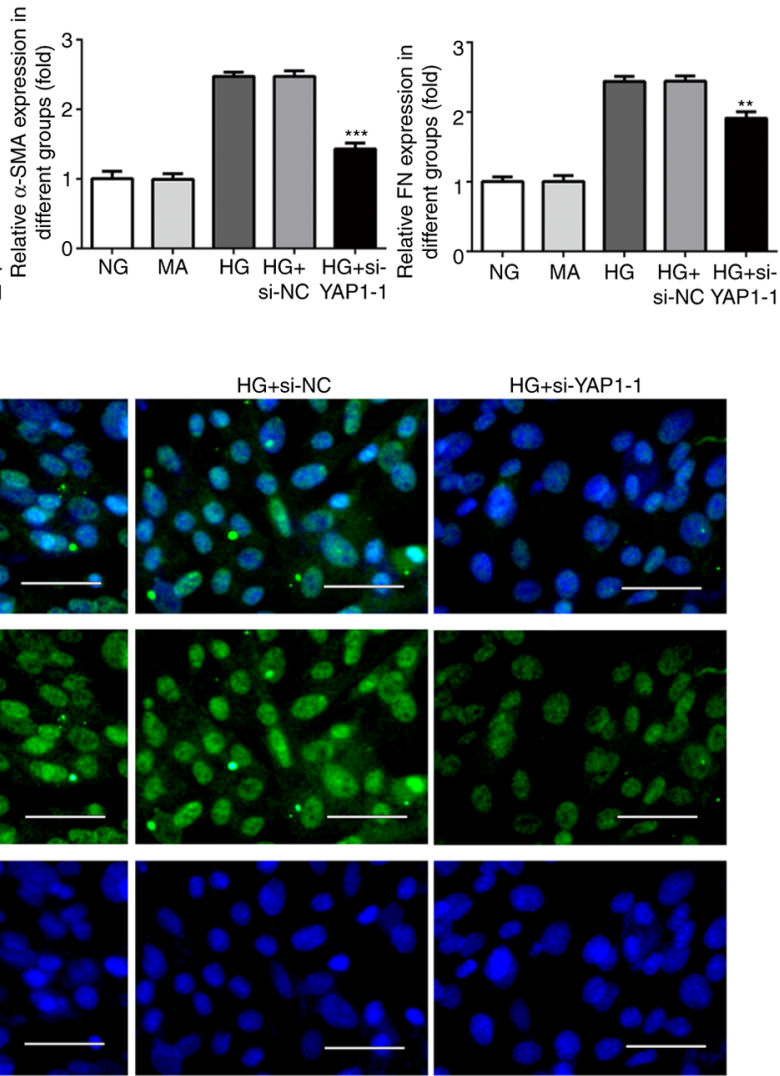
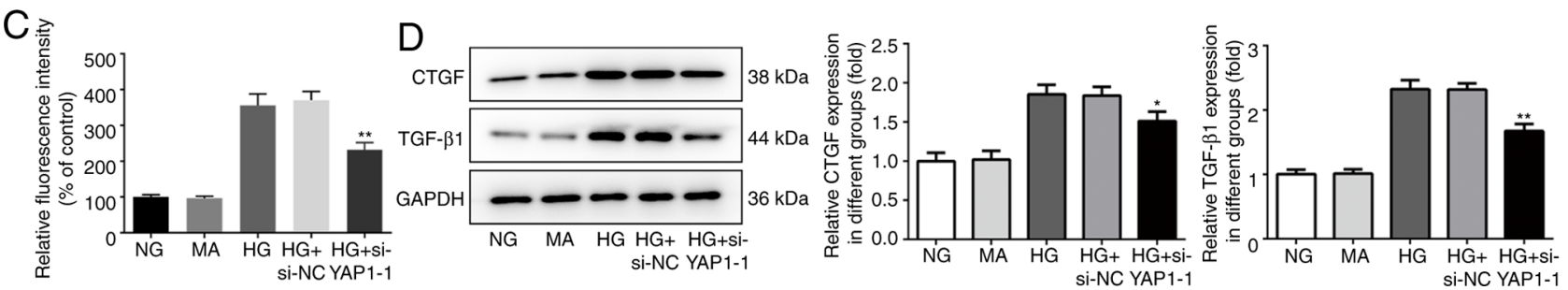

Figure 4. Knockdown of YAP1 inhibits the deposition of extracellular matrix and fibrosis in HBZY-1 cells induced by high glucose. (A) Effect of YAP1 knockdown on the expressions of collagen IV, $\alpha$-SMA and FN was detected by western blotting. (B and C) Effect of YAP1 knockdown on the expressions of collagen IV was detected by immunofluorescence. Scale bars, $50 \mu \mathrm{m}$. (D) Effect of YAP1 knockdown on the expressions of fibrogenic factors CTGF and TGF- $\beta 1$ was detected by western blotting. ${ }^{*} \mathrm{P}<0.05,{ }^{* *} \mathrm{P}<0.01$ and ${ }^{* * *} \mathrm{P}<0.001$ vs. HG + si-NC. YAP1, Yes-associated protein 1 ; si-, small interfering RNA; NC, negative control; HG, high-glucose group; NG, normal control group; MA, mannitol; SMA, smooth muscle actin; FN, fibronectin; CTGF, connective tissue growth factor; TGF, transforming growth factor.

that YAP1 knockdown significantly suppressed the activation of the NF- $\kappa \mathrm{B} / \mathrm{JMJD} 3$ signaling pathway.

Inhibitors of the $N F-\kappa B / J M J D 3$ signaling pathway attenuate the promoting effects of YAP1 on the proliferation and inflammation of HBZY-1 cells treated with high glucose. Next, YAP1 overexpression plasmid was constructed to increase YAP1 expression in HBZY-1 cells (Fig. 6A). NF- $\kappa$ B inhibitor JSH23 and JMJD3 inhibitor GSK-J4 were added to the cells to inhibit the NF- $\mathrm{B} / \mathrm{JMJD} 3$ signaling pathway. As shown in Fig. 6B, JSH23 and GSK-J4 significantly decreased the phosphorylation levels of $\mathrm{I} \kappa \mathrm{B} \alpha$ and $\mathrm{p} 65$, as well as JMJD3 expression. In addition, the cell proliferation results showed that JSH23 and GSK-J4 could partially reverse the promoting effect of YAP1 overexpression on cell viability and MCP-1 expression (Fig. 6C and D). ELISA and western blot analysis results showed that JSH23 and GSK-J4 decreased the expression of TNF- $\alpha$, IL-1 $\beta$, IL-6 and MCP-1.
Inhibitors of the $N F-\kappa B / J M J D 3$ signaling pathway attenuate the promoting effects of YAPI on ECM deposition and fibrosis of high-glucose-treated HBZY-1 cells. Finally, western blot analysis and immunofluorescence assays were performed to determine the effect of YAP1 on ECM deposition and fibrosis of HBZY-1 cells treated with high glucose. As shown in Fig. 7A-C, YAP1 knockdown significantly promoted the expression of collagen IV, $\alpha$-SMA and FN. Similarly, as shown in Fig. 7D, YAP1 knockdown significantly promoted the expression of CTGF and TGF- $\beta 1$. These changes could be partially reversed by the $\mathrm{NF}-\kappa \mathrm{B} / \mathrm{JMJD} 3$ signaling pathway inhibitors JSH23 and GSK-J4.

\section{Discussion}

DN is a microvascular complication of late-stage diabetes and the main cause of ESRD (16). There is currently a lack of sensitive indicators for the early diagnosis of DN; therefore, 
A

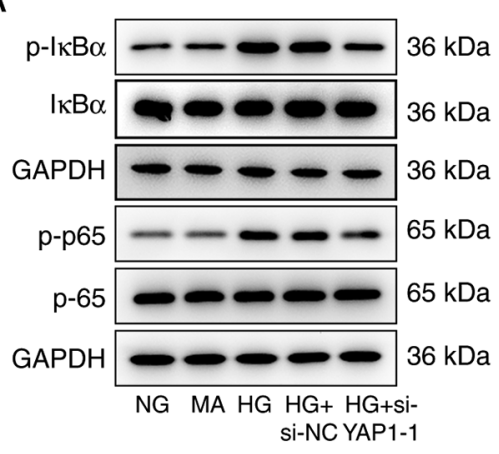

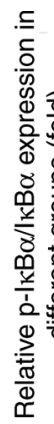
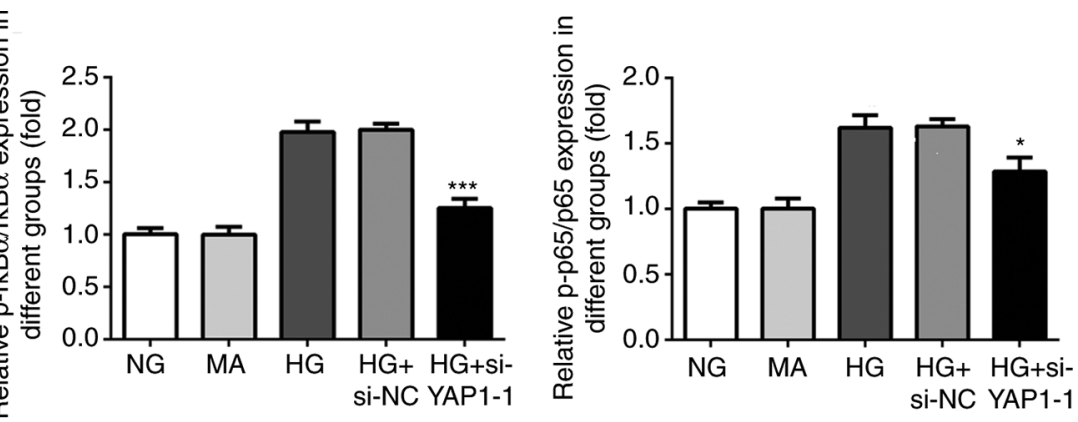

B

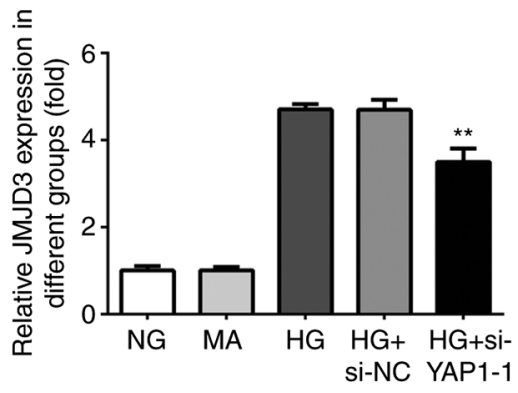

Figure 5. Knockdown of YAP1 inhibited NF-кB/JMJD3 signal pathway in HBZY-1 cells induced by high glucose. (A) Effect of YAP1 knockdown on the

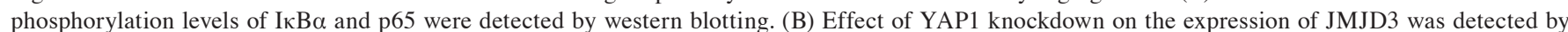
western blotting. ${ }^{*} \mathrm{P}<0.05,{ }^{* *} \mathrm{P}<0.01$ and ${ }^{* * * *} \mathrm{P}<0.001$ vs. $\mathrm{HG}+$ si-NC. YAP1, Yes-associated protein 1; si-, small interfering RNA; NC, negative control; HG, high-glucose group; NG, normal control group; MA, mannitol; NF- $\kappa$ B, nuclear factor- $\kappa$ B; JMJD3, Jumonji domain-containing protein D3; p-, phosphorylated-; $\mathrm{I} \kappa \mathrm{B} \alpha$, inhibitor of $\mathrm{NF}-\kappa \mathrm{B}$.

identifying new diagnostic markers for DN is crucial. The results of the present study revealed that YAP1 was upregulated in HBZY-1 cells treated with high glucose. YAP1 could promote the proliferation, inflammation, ECM deposition and fibrosis of HBZY-1 cells by activating the NF- $\kappa$ B/JMJD3 pathway.

YAP transcriptional coactivator is a key effector of the Hippo signaling pathway and plays an important role in regulating cell proliferation, differentiation and tissue homeostasis (17). YAP has been shown to play an important role in DN. It is highly expressed in renal tissue and associated with the levels of creatinine and blood urea nitrogen, DN stage and pathological grade, which suggests that it plays an important role in renal injury in type 2 DM (18). In addition, YAP overexpression has been found to be closely associated with ECM and fibrosis (10). YAP1 knockdown could significantly decrease the deposition of ECM and improve pulmonary fibrosis (13). YAP1 has been shown to promote tumor growth in both in vivo and in vitro experiments. In combination with TEAD, YAP1 could activate the transcription of IL-1 $\beta$, a key inflammatory cytokine in gastric cells, eventually leading to gastric cancer, since IL-1 $\beta$ can induce inflammation-associated tumorigenesis. Therefore, YAP1 can play a key role in increasing inflammation by activating inflammatory cytokines (14). It was recently demonstrated that YAP1 silencing may have a therapeutic effect on diabetic retinopathy (19). Since excessive proliferation of GMCs and ECM deposition are the main pathological characteristics associated with the occurrence and development of DN $(20,21)$, it was hypothesized that YAP1 may promote the inflammation and ECM deposition of GMCs induced by high glucose. In the present study, YAP1 was upregulated in HBZY-1 cells treated with high glucose. YAP1 knockdown significantly suppressed the proliferation, inflammation, ECM deposition and fibrosis of HBZY-1 cells treated with high glucose. These results highlighted the importance of YAP1 in the process of DN.

To further investigate the specific regulatory mechanism of YAP1 in DN, a large body of studies was used to screen the signaling pathways that may interact with YAP1. JMJD3, also known as lysine demethylase 6B, is located on human chromosome 17. Its abnormal expression is closely associated with the occurrence and development of various types of cancer, and it is considered to be a tumor evaluation index with a potentially great application value $(22,23)$. A previous study showed that JMJD3 significantly promoted IL-1 $\beta$ expression in the early stage of sepsis (24). In addition, JMJD3 may regulate neointimal proliferation following vascular injury and promote joint destruction in rheumatoid arthritis by promoting the proliferation and migration of fibroblast-like synoviocytes (25). As the upstream target of JMJD3, NF- $\kappa$ B regulates inflammatory gene expression in vascular endothelial cells (26). In addition, cystitis promotes NF- $\kappa$ B pathway activation and JMJD3 expression, thereby inducing proliferation of human bladder smooth muscle cells and ECM deposition (27). It is worth noting that YAP1 was shown to inhibit the negative regulatory factor of NF- $\kappa \mathrm{B}$ USP31 and induce excessive tumor cell proliferation. The aberrant stabilization of YAP1 has been shown to significantly enhance the activity of the $\mathrm{NF}-\kappa \mathrm{B}$ pathway (28). Therefore, it was hypothesized that YAP1 may activate the NF- $\kappa \mathrm{B} / \mathrm{JMJD} 3$ signaling pathway and promote inflammation and ECM deposition in HBZY-1 cells treated with high glucose. In the present study, the expression 
B

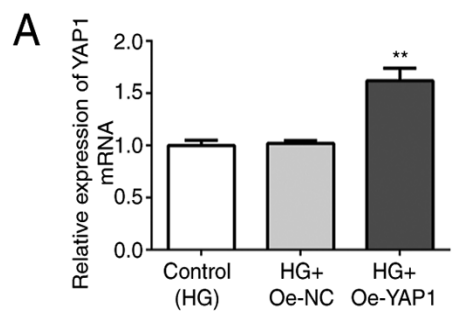

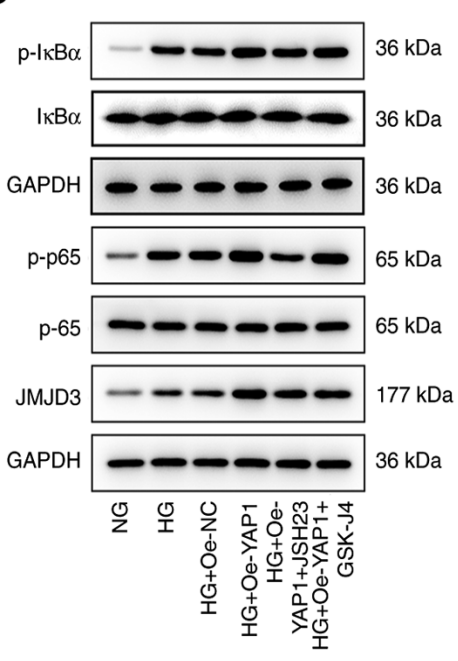
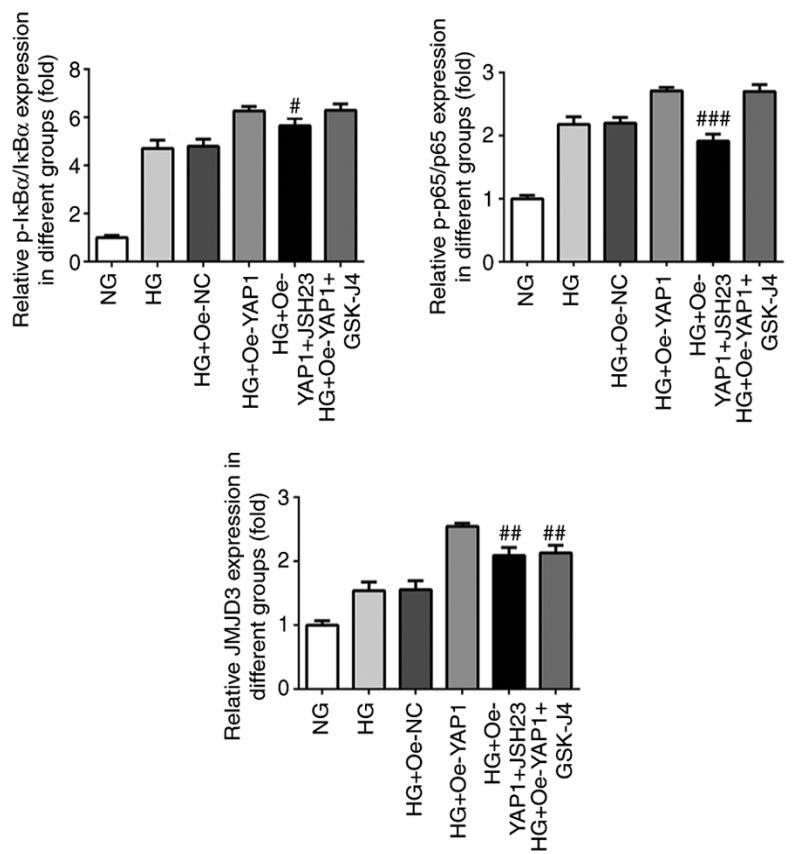

C

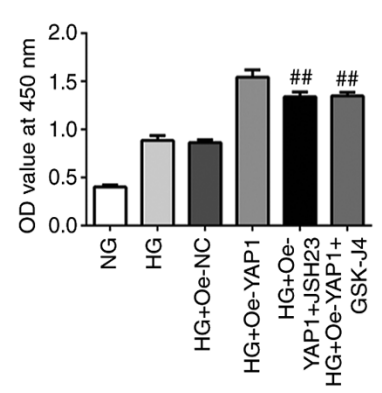

D
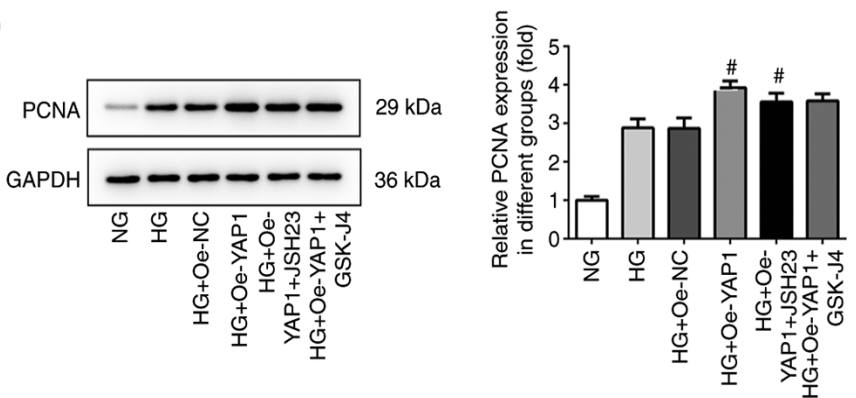

E
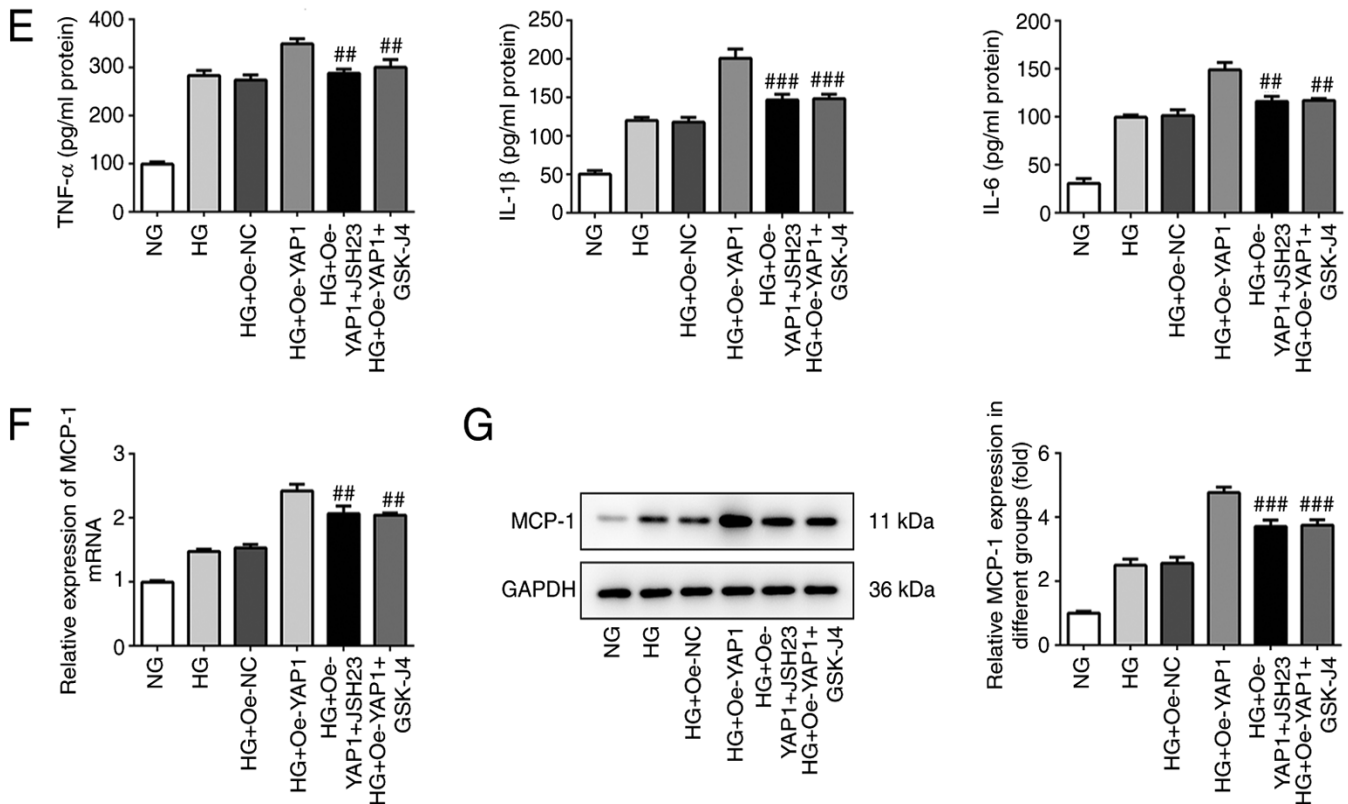

G

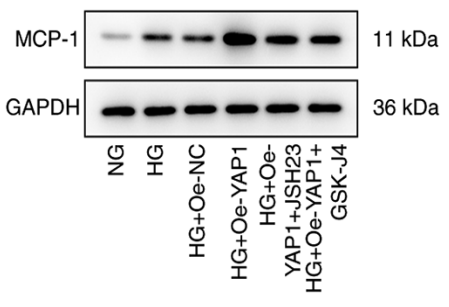

Figure 6. Inhibitors of NF-kB/JMJD3 signal pathway attenuates the facilitatory effects of YAP1 on the proliferation and inflammation of HBZY-1 cells induced by high glucose. (A) The efficiency of YAP1 overexpression was determined by RT-qPCR. (B) Effect of the inhibitors of NF-kB/JMJD3 signal pathway on the expression levels of IkB $\alpha$ and $p 65$ were detected by western blotting. (C) Effect of the inhibitors of NF- $\mathrm{kB} / \mathrm{JMJD} 3$ signal pathway on the viability of HBZY-1 cells treated with high glucose was detected by Cell Counting Kit-8 assay. (D) Effect of the inhibitors of NF-kB/JMJD3 signal pathway on the expression of PCNA in the HBZY-1 cells treated with high glucose was measured by western blotting. (E) Effect of the inhibitors of NF-кB/JMJD3 signal pathway on the expressions of pro-inflammatory factors (TNF- $\alpha$, IL-1 $\beta$ and IL-6) was detected by enzyme-linked immunosorbent assay. (F) Effect of the inhibitors of NF-кB/JMJD3 signal pathway on the expression of MCP-1 was detected by RT-qPCR. (G) Effect of the inhibitors of NF- $\mathrm{kB} / \mathrm{JMJD} 3$ signal pathway on the expression of MCP-1 was detected by western blotting. ${ }^{* *} \mathrm{P}<0.01$ vs. $\mathrm{HG}+\mathrm{Oe}-\mathrm{NC} .{ }^{\#} \mathrm{P}<0.05,{ }^{\# \#} \mathrm{P}<0.01$ and ${ }^{\# \# \#} \mathrm{P}<0.001$ vs. HG + Oe-YAP1. YAP1, Yes-associated protein 1; RT-qPCR, reverse transcription-quantitative PCR; Oe, overexpression vector; NC, negative control; HG, high-glucose group; NG, normal control

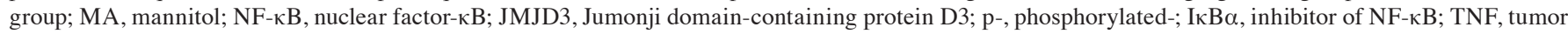
necrosis factor; IL, interleukin; MCP-1, monocyte chemoattractant protein-1. 

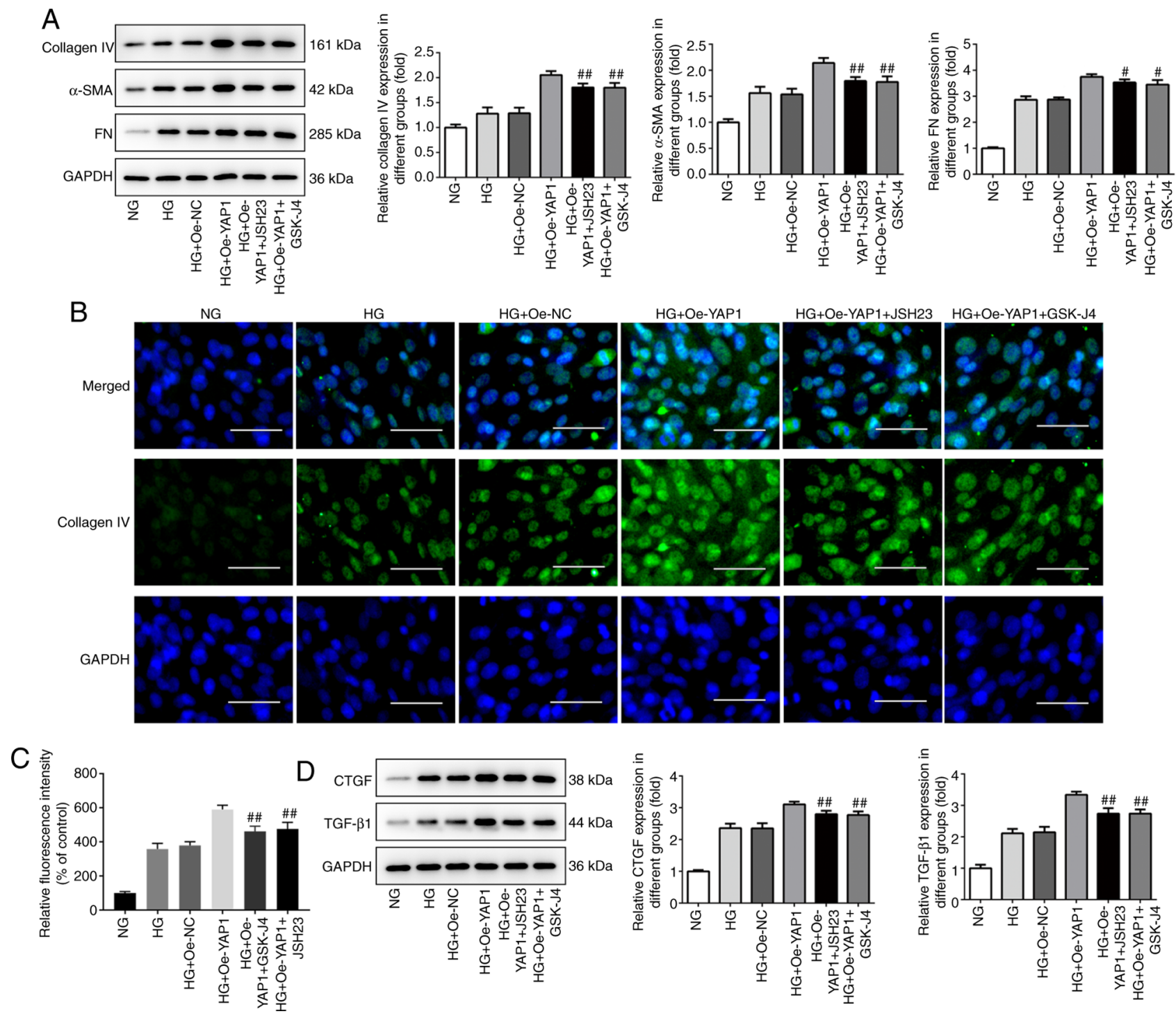

Figure 7. Inhibitors of NF- $\mathrm{kB} / \mathrm{JMJD} 3$ signal pathway attenuates the facilitatory effects of YAP1 on the deposition of extracellular matrix and fibrosis of HBZY-1 cells induced by high glucose. (A) Effect of the inhibitors of NF- $\mathrm{kB} / \mathrm{JMJD} 3$ signal pathway on the expression levels of collagen IV, $\alpha$-SMA and FN was detected by western blotting. (B and C) Effect of the inhibitors of NF-kB/JMJD3 signal pathway on the expression levels of collagen IV was

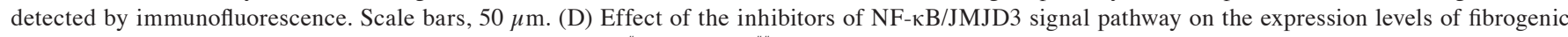
factors CTGF and TGF- $\beta 1$ was detected by western blotting. " $\mathrm{P}<0.05$ and ${ }^{\# \#} \mathrm{P}<0.01$ vs. HG + Oe-YAP1. YAP1, Yes-associated protein 1 ; Oe, overexpression

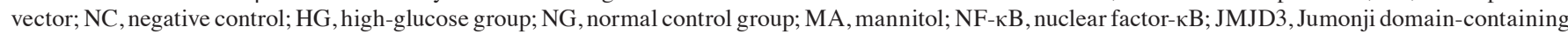
protein D3; SMA, smooth muscle actin; FN, fibronectin; CTGF, connective tissue growth factor; TGF, transforming growth factor.

of $\mathrm{NF}-\kappa \mathrm{B} / \mathrm{JMJD} 3$ signaling pathway-associated proteins was upregulated in HBZY-1 cells treated with high glucose, and inhibitors of $\mathrm{NF}-\kappa \mathrm{B} / \mathrm{JMJD} 3$ signaling could significantly enhance the promoting effect of YAP1 on inflammation and ECM deposition of HBZY-1 cells treated with high glucose.

YAP-1 is a well-characterized downstream transcriptional co-activator of Hippo pathway that interacts with various transcription factors and modulates their transcriptional activities. However, the association between Hippo pathway and YAP-1-induced inflammation and deposition of extracellular matrix in diabetic nephropathy was not study. In addition, it was further explored how YAP-1 could potentially activate NF- $\kappa$ B. The aforementioned issues will be studied in further research.

In conclusion, YAP1 may aggravate high glucose-induced GMC inflammation and ECM deposition by activating the $\mathrm{NF}-\kappa \mathrm{B} / \mathrm{JMJD} 3$ signaling pathway. The results of the present study may indicate a new direction for the identification of therapeutic targets for DN.

\section{Acknowledgements}

Not applicable.

\section{Funding}

This work was supported by the Fundamental Research Funds for the Provincial Universities (grant no. 2019-KYYWF-0373).

\section{Availability of data and materials}

All data generated or analyzed during this study are included in this published article. 


\section{Authors' contributions}

YW and ZC designed the experiments and made considerable contributions to the manuscript writing. YW and JX performed the experiments and analyzed the data. $\mathrm{ZC}$ revised the manuscript and guided the experiments. ZC and JX confirm the authenticity of all the raw data. All authors read and approved the final manuscript.

\section{Ethical approval and consent to participate}

Not applicable.

\section{Patient consent for publication}

Not applicable.

\section{Competing interests}

The authors declare that they have no competing interests.

\section{References}

1. Yuan CM, Nee R, Ceckowski KA, Knight KR and Abbott KC: Diabetic nephropathy as the cause of end-stage kidney disease reported on the medical evidence form CMS2728 at a single center. Clin Kidney J 10: 257-262, 2017.

2. Duran-Salgado MB and Rubio-Guerra AF: Diabetic nephropathy and inflammation. World J Diabetes 5: 393-398, 2014.

3. Tessari P: Nitric oxide in the normal kidney and in patients with diabetic nephropathy. J Nephrol 28: 257-268, 2015.

4. Wada $J$ and Makino H: Inflammation and the pathogenesis of diabetic nephropathy. Clin Sci (Lond) 124: 139-152, 2013.

5. Meza Letelier CE, San Martín Ojeda CA, Ruiz Provoste JJ and Frugone Zaror CJ: Pathophysiology of diabetic nephropathy: A literature review. Medwave 17: e6839, 2017 (In Spanish).

6. Xu X, Xiao L, Xiao P, Yang S, Chen G, Liu F, Kanwar YS and Sun L: A glimpse of matrix metalloproteinases in diabetic nephropathy. Curr Med Chem 21: 3244-3260, 2014.

7. Kwiatkowska E, Domanski L, Bober J, Safranow K Romanowski M, Pawlik A, Kwiatkowski S and Ciechanowski K: Urinary metalloproteinases-9 and-2 and their inhibitors TIMP-1 and TIMP-2 are markers of early and long-term graft function after renal transplantation. Kidney Blood Press Res 41: 288-297, 2016.

8. Mazanowska O, Żabińska M, Kościelska-Kasprzak K, Kamińska D, Krajewska M, Banasik M, Madziarska K, Zmonarski SC, Chudoba P, Biecek P, et al: Increased plasma matrix metalloproteinase-2 (MMP-2), tissue inhibitor of proteinase-1 (TIMP-1), TIMP-2, and urine MMP-2 concentrations correlate with proteinuria in renal transplant recipients. Transplant Proc 46: 2636-2639, 2014.

9. Hu K, Mars WM and Liu Y: Novel actions of tissue-type plasminogen activator in chronic kidney disease. Front Biosci 13: 5174-5186, 2008

10. Li FJ, Surolia R, Li H, Wang Z, Liu G, Liu RM, Mirov SB, Athar M, Thannickal VJ and Antony VB: Low-dose cadmium exposure induces peribronchiolar fibrosis through site-specific phosphorylation of vimentin. Am J Physiol Lung Cell Mol Physiol 313: L80-L91, 2017.

11. Grampa V, Delous M, Zaidan M, Odye G, Thomas S, Elkhartoufi N, Filhol E, Niel O, Silbermann F, Lebreton C, et al: Novel NEK8 mutations cause severe syndromic renal cystic dysplasia through YAP dysregulation. PLoS Genet 12: e1005894, 2016.

12. Rinschen MM, Grahammer F, Hoppe AK, Kohli P, Hagmann H, Kretz O, Bertsch S, Höhne M, Göbel H, Bartram MP, et al: YAP-mediated mechanotransduction determines the podocyte's response to damage. Sci Signal 10: eaaf8165, 2017.
13. Chen Y, Zhao X, Sun J, Su W, Zhang L, Li Y, Liu Y, Zhang L, Lu Y, Shan $\mathrm{H}$ and Liang H: YAP1/Twist promotes fibroblast activation and lung fibrosis that conferred by miR-15a loss in IPF. Cell Death Differ 26: 1832-1844, 2019.

14. Wu Y, Shen L, Liang X, Li S, Ma L, Zheng L, Li T, Yu H, Chan H, Chen $\mathrm{C}$, et al: Helicobacter pylori-induced YAP1 nuclear translocation promotes gastric carcinogenesis by enhancing IL-1 $\beta$ expression. Cancer Med 8: 3965-3980, 2019.

15. Wang GL, Xia XL, Li XL, He FH and Li JL: Identification and expression analysis of the MSP130-related-2 gene from Hyriopsis cumingii. Genet Mol Res 14: 4903-4913, 2015.

16. Mulder S, Hamidi H, Kretzler M and Ju W: An integrative systems biology approach for precision medicine in diabetic kidney disease. Diabetes Obes Metab 20 (Suppl 3): S6-S13, 2018.

17. Choi SY, Bae H, Jeong SH, Park I, Cho H, Hong SP, Lee DH, Lee CK, Park JS, Suh SH, et al: YAP/TAZ direct commitment and maturation of lymph node fibroblastic reticular cells. Nat Commun 11: 519, 2020

18. Ma R, Ren JM, Li P, Zhou YJ, Zhou MK, Hu Z and Xiao XY: Activated YAP causes renal damage of type 2 diabetic nephropathy. Eur Rev Med Pharmacol Sci 23: 755-763, 2019.

19. Han N, Tian W, Yu N and Yu L: YAP1 is required for the angiogenesis in retinal microvascular endothelial cells via the inhibition of MALAT1-mediated miR-200b-3p in high glucoseinduced diabetic retinopathy. J Cell Physiol 235: 1309-1320, 2020.

20. Park MJ, Kim DI, Lim SK, Choi JH, Han HJ, Yoon KC and Park SH: High glucose-induced O-GlcNAcylated carbohydrate response element-binding protein (ChREBP) mediates mesangial cell lipogenesis and fibrosis: The possible role in the development of diabetic nephropathy. J Biol Chem 289: 13519-13530, 2014.

21. Wang J, Yang Q, Nie Y, Guo H, Zhang F, Zhou X and Yin X: Tetrahydrobiopterin contributes to the proliferation of mesangial cells and accumulation of extracellular matrix in early-stage diabetic nephropathy. J Pharm Pharmacol 69: 182-190, 2017.

22. Sakaki H, Okada M, Kuramoto K, Takeda H, Watarai H, Suzuki S, Seino S, Seino M, Ohta T, Nagase S, et al: GSKJ4, a selective jumonji H3K27 demethylase inhibitor, effectively targets ovarian cancer stem cells. Anticancer Res 35: 6607-6614, 2015.

23. Tokunaga R, Sakamoto Y, Nakagawa S, Miyake K, Izumi D, Kosumi K, Taki K, Higashi T, Imamura Y, Ishimoto T, et al: The prognostic significance of histone lysine demethylase JMJD3/KDM6B in colorectal cancer. Ann Surg Oncol 23: 678-685, 2016.

24. Chen Y, Liu Z, Pan T, Chen E, Mao E, Chen Y, Tan R, Wang X, Tian R, Liu J and Qu H: JMJD3 is involved in neutrophil membrane proteinase 3 overexpression during the hyperinflammatory response in early sepsis. Int Immunopharmacol 59: 40-46, 2018.

25. Jia W, Wu W, Yang D, Xiao C, Su Z, Huang Z, Li Z, Qin M, Huang M, Liu S, et al: Histone demethylase JMJD3 regulates fibroblast-like synoviocyte-mediated proliferation and joint destruction in rheumatoid arthritis. FASEB J 32: 4031-4042, 2018.

26. Yu S, Chen X, Xiu M, He F, Xing J, Min D and Guo F: The regulation of Jmjd 3 upon the expression of NF- $\kappa$ B downstream inflammatory genes in LPS activated vascular endothelial cells. Biochem Biophys Res Commun 485: 62-68, 2017.

27. Lai J, Ge M, Shen S, Yang L, Jin T, Cao D, Xu H, Zheng X, Qiu S, Wang K, et al: Activation of NFKB-JMJD3 signaling promotes bladder fibrosis via boosting bladder smooth muscle cell proliferation and collagen accumulation. Biochim Biophys Acta Mol Basis Dis 1865: 2403-2410, 2019.

28. Ye S, Lawlor MA, Rivera-Reyes A, Egolf S, Chor S, Pak K, Ciotti GE, Lee AC, Marino GE, Shah J, et al: YAP1-mediated suppression of USP31 enhances NFKB activity to promote sarcomagenesis. Cancer Res 78: 2705-2720, 2018.

This work is licensed under a Creative Commons Attribution-NonCommercial-NoDerivatives 4.0 International (CC BY-NC-ND 4.0) License. 